\title{
Concurrence of rheumatoid arthritis and systemic lupus erythematosus: report of 11 cases
}

\author{
MICHAEL G COHEN AND JOHN WEBB \\ From the Sydney University Rheumatology Department, The Royal North Shore Hospital of Sydney, St \\ Leonards, NSW 2065, Australia
}

SUMMARY The concurrence of rheumatoid arthritis (RA) and systemic lupus erythematosus (SLE) has been reported infrequently. Eleven patients are described here with both RA and SLE, in whom the diagnoses were separated by one to 24 years. Because of the difficulty in diagnosing RA occurring subsequent to SLE, only patients with classical RA as their initial diagnosis were included. Further difficulties arise because arthritis is common to both diseases and may be deforming in SLE, antinuclear antibodies (ANA) are not uncommon in RA, and rheumatoid factor (RF) may be seen in SLE. Nonetheless, judicious application of the American Rheumatism Association (ARA) criteria allows both diagnoses to be made in the individual patient. In our patients there was erosive arthritis in nine, rheumatoid nodules in five, and urinary abnormalities in 10 . Serological evidence of RA and SLE with positive RF and ANA and raised DNA antibodies was universal, all patients had haematological evidence of SLE, and all but one decreased serum complement levels. These cases suggest that the concurrence of RA and SLE is not as rare as previously considered and may occur more often than expected by chance alone.

Key words: overlap syndromes, antinuclear antibodies, rheumatoid factor.

Despite the clinical and laboratory similarities of rheumatoid arthritis (RA) and systemic lupus erythematosus (SLE) distinction between the two diseases can usually be made, though at times this may be difficult. It might also be expected that some patients would have both diseases, but the paucity of such reported instances has led to the suggestion that the concurrence of RA and SLE is coincidental. ${ }^{1}$ Were this so, however, about $1 \%$ of patients with SLE would be expected to have concurrent RA as this represents the prevalence of definite or classical RA in most communities. ${ }^{2}$ If the small numbers of published cases truly reflect the prevalence of associated RA and SLE then it would imply that there is a negative association between these diseases. ${ }^{3}$ Given the clinical and serological overlap between RA and SLE, this seems unlikely, and thus the apparent under-reporting probably reflects the difficulty of making both diagnoses in the individual patient. Criteria for the diagnosis of

Accepted for publication 10 May 1987.

Correspondence to Dr Michael G Cohen, Sydney University Rheumatology Department. The Royal North Shore Hospital of Sydney, St Leonards, NSW 2065, Australia.
SLE have been defined and updated. ${ }^{4}$ On the other hand, the diagnostic criteria of $\mathrm{RA}^{5}$ have remained unchanged since 1958 and contain the specific exclusion of SLE.

We presently report 11 patients with classical RA who subsequently developed SLE. We believe that these cases probably underestimate the true prevalence of concurrence of the two diseases in our patient population. We describe these patients to support the notion that RA and SLE may be concurrent, and to show the diagnostic difficulties that may arise.

\section{Patients and methods}

All patients attending the rheumatology department at the Royal North Shore Hospital of Sydney during the past 17 years who had the dual diagnoses of RA and SLE entered separately or simultaneously were reviewed.

The hospital is a secondary referral institution for the lower north shore of Sydney (population $160000)$ and a tertiary referral centre for the northern region of New South Wales (population 800000 ). Because of the specialised interest that we 
have in SLE, patients having this syndrome are more likely to be referred to us than those having other rheumatic complaints, including RA. During the past 17 years 309 patients have been seen in our unit in whom SLE has been diagnosed.

Only those who fulfilled the ARA criteria, firstly, for classical $\mathrm{RA}^{5}$ and, subsequently, for $\mathrm{SLE}^{4}$ (excluding polyarthritis) were further studied. Because of the occurrence of nodules, ${ }^{6}$ rheumatoid factor (RF) ${ }^{7}$ and, rarely, erosions ${ }^{8}$ in SLE we have not included those patients in whom the initial diagnosis was SLE. Also, no patient having clinical or laboratory features (abnormal urine sediment, raised levels of DNA antibodies, or hypocomplementaemia) to suggest SLE at the time of diagnosis of RA was included in this study.
Careful review of the case records was supplemented by current clinical evaluation, serology, and joint radiographs of all surviving patients. All serology, during the period reviewed, has been done in our laboratory without any major changes in technological procedures. Estimation of RF titre was by a commercial sensitised sheep cell agglutination technique (Rheumaton, Denver Laboratories). Antinuclear antibody (ANA) was detected using rat is liver substrate as previously described. ${ }^{9}$ Native- $\vec{\circ}$ DNA antibody (anti-DNA) was measured as percentage binding in the Farr assay, with the upper level of normal being $20 \%{ }^{10}$ Antibodies to extractable nuclear antigens (anti-ENA) were detected by counterimmunoelectrophoresis using rabbit thymus of (Pel Freeze Biologicals, AR, USA) and guinea pig

Table 1 Clinical and laboratory details

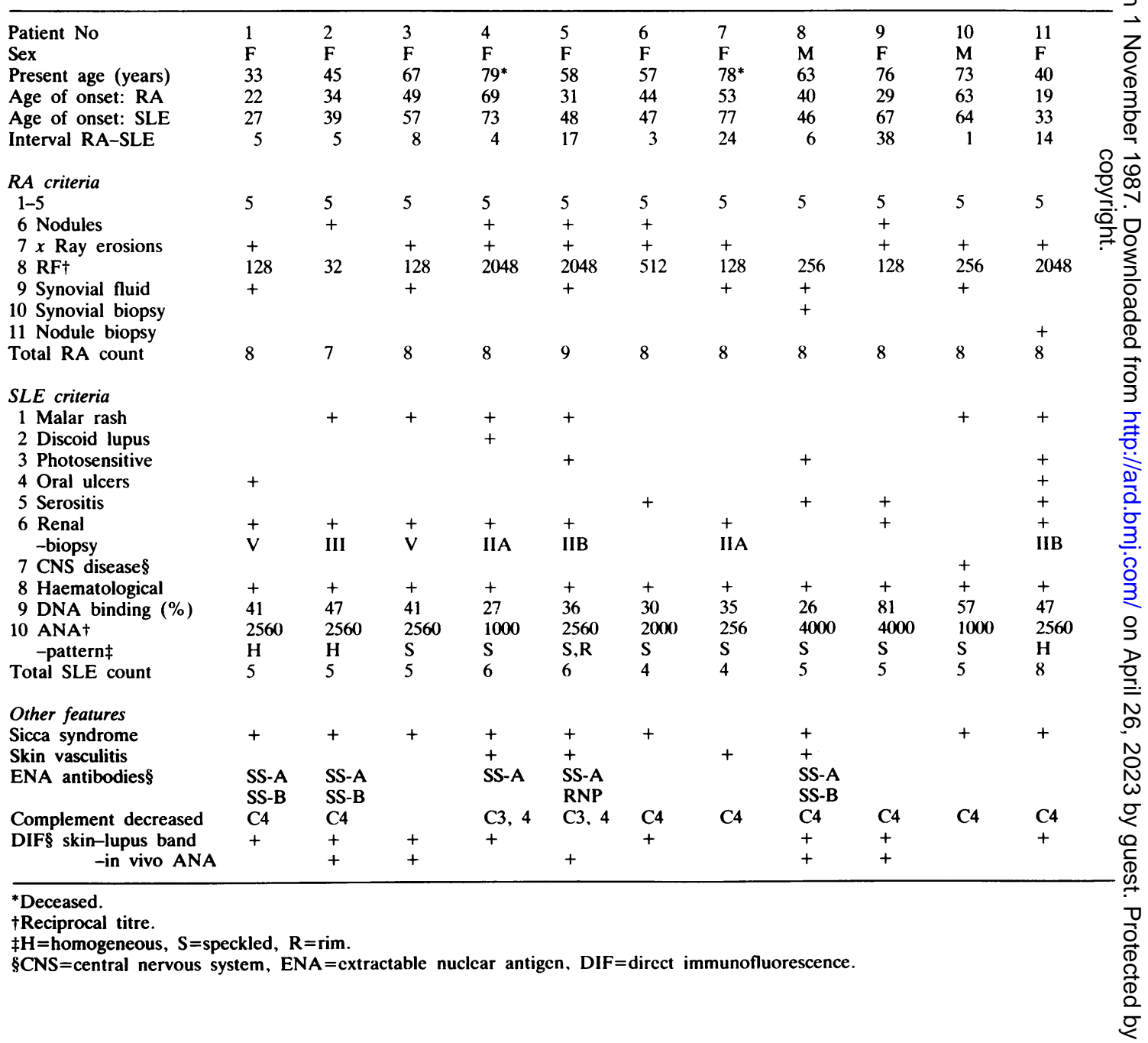


kidney (Sigma Chemicals, St Louis, MO, USA) extracts ${ }^{9}$ and identified by immunodiffusion against known reference sera in agarose gels. Synovial fluid analysis was by standard techniques. Renal biopsy specimens were assessed by light and electron microscopy and by direct immunofluorescence and categorised according to the WHO classification. ${ }^{11}$ Skin biopsy specimens were obtained from uninvolved skin on the inner aspect of the upper arm and examined by light microscopy and direct immunofluorescence. ${ }^{9}$

\section{Results}

CLINICAL AND LABORATORY FINDINGS The ages of onset of RA and SLE, the ARA criteria for the two diseases ${ }^{45}$ fulfilled by each patient, and other clinical and laboratory findings are shown in Table 1 . The mean age of onset of RA was $41 \cdot 2$ years (range 19-69) and that of SLE was 52.5 years (range 27-77), giving a mean interval between the diagnoses of the two diseases of 11.3 years (range 1-38). The mean follow up from the diagnosis of SLE has been $8 \cdot 2$ years (range 1-17).

\section{CHARACTERISTICS OF RHEUMATOID}

A R T H R I T IS

All patients had morning stiffness, joint pain and tenderness, and symmetrical polyarthritis, thus fulfilling criteria one to five for RA. Similarly, a positive RF was detected in all patients and was, with the exception of patient No 2, in moderate or high titre. Erosive arthritis was seen in nine patients and in eight it was deforming. Among the patients with the greatest deformity, a spectrum of mild (Fig. 1) to marked erosive disease was observed. Of the five patients with rheumatoid nodules, only patient No 11 had biopsy verification. Synovial fluid analysis was performed in six patients and was consistent with RA in all, including a synovial RF titre equal to or higher than that found in the corresponding sera. Each of the patients had at least seven criteria (mean eight) and may thus be classified as having classical RA.

CHARACTERISTICS OF SYSTEMIC LUPUS ER Y T HEM A T OS US

The onset of SLE was most commonly marked by the detection of urinary abnormalities, and seven patients had renal biopsies, all of which were consistent with lupus glomerulonephritis (LGN). Only patients Nos 2 and 3 required cytotoxic therapy for renal involvement. Malar rash was common, being present in six patients. All patients had haematological abnormalities that could not be ascribed to drug therapy; lymphopenia $\left(<10^{9} / 1\right)$ was present in all, leucopenia $\left(<4 \times 10^{9} / 1\right)$ in four (patients 1, 3, 5, and 10), thrombocytopenia in patient No $6\left(40 \times 10^{9} / 1\right)$, and four had positive direct Coombs' tests (patients 1,2,10, and 11). Partial thromboplastin times with kaolin were normal in the 10 patients in which it was sought. High titre ANA positivity and raised anti-DNA were a universal finding. The patients had a mean of $5 \cdot 3$ criteria for SLE, with the exclusion of arthritis.

As D-penicillamine is associated with drug related

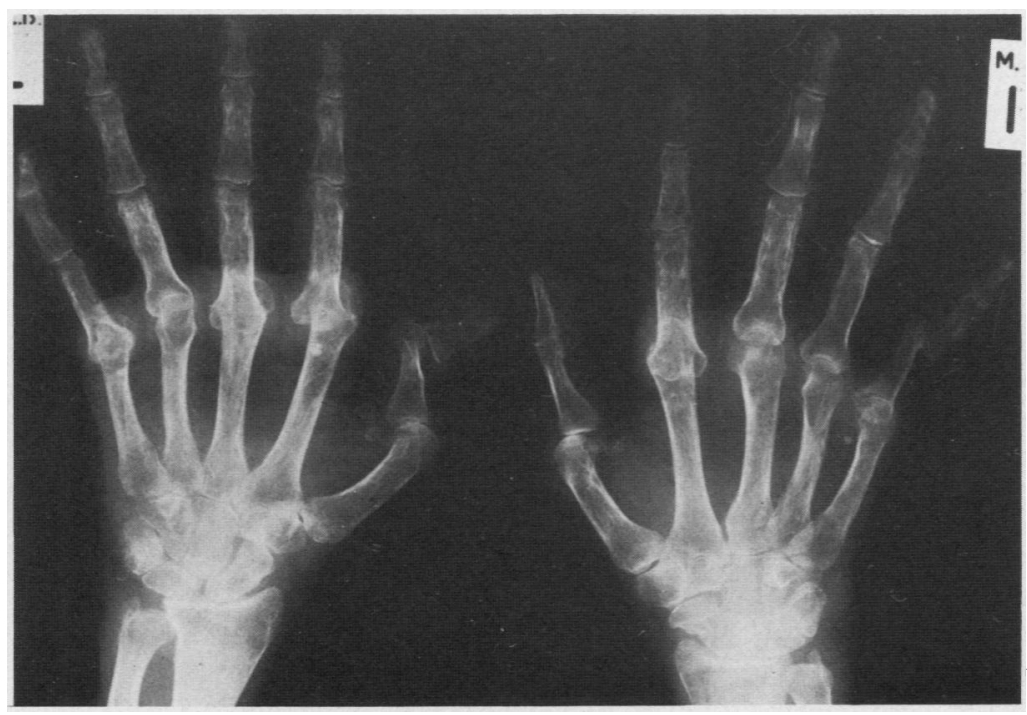

Fig. 1 Patient No 5. There is osteopenia and obvious deformity with subluxation or dislocation involving several metacarpophalangeal (MCP) joints, the interphalangeal joint of the left thumb, and the right fifth proximal interphalangeal (PIP) joint. Relatively minor erosions involve a number of the MCP and PIP joints. 


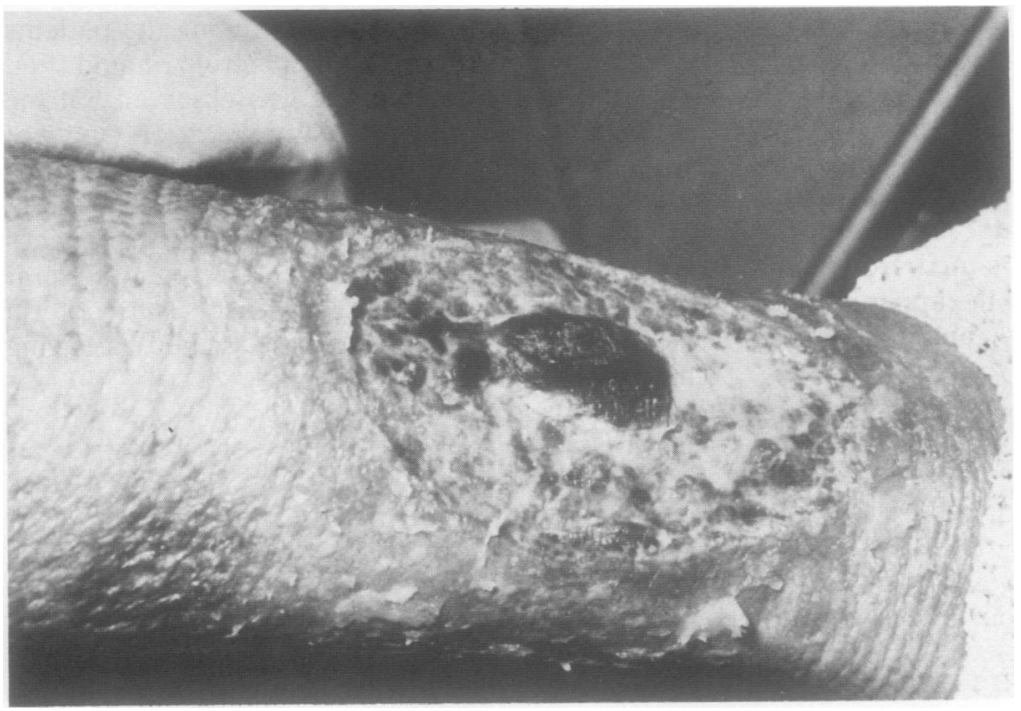

Fig. 2 Patient No 8. A deep necrotic ulcer is apparent on the lower leg.

lupus erythematosus and is unique in that it will induce antibodies to double stranded $D_{N A}{ }^{12} 13$ we reviewed the possible relation between $D$ penicillamine and the development of SLE in the two patients that received it. Patient No 1 had oral ulceration that preceded the administration of $\mathrm{D}$ penicillamine, and the serological abnormalities together with the renal biopsy showing membranous LGN were found a year after this drug was stopped owing to lack of efficacy. Patient No 2 developed the nephrotic syndrome, which was due to membranous LGN and settled upon withdrawal of Dpenicillamine, which was not reintroduced. Two years later, however, she developed malar rash and decreasing renal function associated with increased numbers of urinary casts. Renal biopsy showed a focal and segmental proliferative LGN with sclerosed glomeruli but no evidence of residual membranous changes.

OTHER DISEASES AND AUTOIMMUNE

FEATURES

Other features in our patients included the sicca syndrome in nine and digital vasculitis or vasculitic leg ulcers (Fig. 2) in four. Anti-ENA were found in five, their specificities being to SS-A and SS-B in three, SS-A and RNP in one, and SS-A alone in another. All but one patient had hypocomplementaemia and only two lacked positive skin biopsy direct immunofluorescence findings ('lupus band' or in vivo ANA, or both).

Significant other diseases were noted in three patients. Four years after the onset of RA patient No 3 developed definite polymyositis, ${ }^{14}$ for which corticosteroids were given and later stopped over a year before the diagnosis of SLE with Sjögren's syndrome. After a further six years, malabsorption with protein losing enteropathy was recorded anc considered secondary to exocrine pancreatic insuffi ciency and amyloidosis which was demonstrated by rectal biopsy. Whether the pancreatic disease is secondary to Sjögren's syndrome or amyloidosis is unknown. Patient No 4 developed a monoclonal $\operatorname{IgM} \varkappa$ paraproteinaemia one year after the onset of SLE and subsequently died of an aggressive immunoblastic lymphoma. Patient No 6 had familial IgA deficiency detected when she was evaluated for RA. She later developed definite dermatomyositis three months before the diagnosis of SLE, and four years later developed Hodgkin's disease, which required treatment with chemotherapy and from which she is currently in remission.

\section{Discussion}

Reports of the concurrence of adult RA and SLE are rare, with Fischman et al finding only five instances in their review of the published work and contributing a further case. ${ }^{1}$ We could find only two other well documented cases, ${ }^{15}$ 16 though 10 cases of 'apparent' juvenile rheumatoid arthritis that progressed to SLE have also been reported. ${ }^{17}$

To obtain a combined diagnosis of RA and SLE is set about by a number of difficulties and therefore we elected to include only those patients who first developed classical RA and subsequently SLE. The criteria for RA lack the specificity to distinguish this disease from many other inflammatory arthritides 
which are exclusions to the diagnosis. ${ }^{5}$ Further, the possibility that $5-23 \%$ of patients with SLE may have a non-erosive, deforming, Jaccoud-type of arthritis $^{18}$ and $21-57 \%$ have positive $\mathrm{RF}^{7}$ complicates the distinction. Two of our patients had non-deforming, non-erosive arthritis. The remaining nine, however, had erosions, thus precluding a diagnosis of Jaccoud-type arthritis, though in patients 5 and 10 the erosive changes were disproportionately minor when compared with the degree of deformity. Erosive changes are rarely accepted as occurring in SLE, ${ }^{719}$ though $5-7 \%$ may have nodules which, histologically, may be similar to rheumatoid nodules. ${ }^{6}$ Importantly, in these 11 patients the initial disease was RA, and its features as exemplified by the classical diagnostic criteria were indistinguishable from those of our other patients with RA. None had clinical features or serological evidence of SLE at the time of diagnosis of classical RA.

It is likely that the diagnosis of SLE can be made with greater assurance. The 1982 criteria for $\mathrm{SLE}^{4}$ allow this disease to be distinguished from a control group of inflammatory arthritides with a high degree of confidence. Notably, most of the control group had RA. When the individual criteria were assessed, arthritis was the least discriminatory. Antinuclear antibody (ANA) was also a poor discriminatory factor as $51 \%$ of the control group and $61 \%$ of our own patients with RA were positive. ${ }^{20}$ Although positive ANA is of little value in distinguishing between RA and SLE, a negative ANA mitigates strongly against the latter diagnosis. Nonetheless, the occurrence of LGN, raised anti-DNA, and hypocomplementaemia, all of which are distinctly uncommon in RA, strongly support the diagnoses of SLE in our patients.

The mean age of onset of SLE in our patients ( 52.5 years) reflects our requirement that RA be the initial diagnosis. Some $12 \%$ of patients with SLE may have their onset of disease in the fifth decade or later, ${ }^{21}$ and such patients usually follow a benign course with a good prognosis. ${ }^{21} 22$ Most of our patients had mild disease with two deaths in the mean follow up period of $8 \cdot 2$ years. Although renal involvement was a feature in eight patients, only two required cytotoxic therapy for LGN. This is consistent with the previous observation that renal involvement tends to be mild in patients with RA and SLE. ${ }^{\prime}$

These 11 patients represent $3.6 \%$ of our patients with SLE, which is greater than the $1 \%$ or less of the population that would be predicted to have classical RA. We consider that some of our reviewed patients with SLE who later developed erosive or nodular arthritis, or both, and became seropositive represent
RA subsequent to SLE, though it may be argued that these are a subgroup of SLE. Thus we believe that the true prevalence of the concurrence of RA and SLE in our patient population has been understated. It seems logical to expect that some patients with SLE would subsequently develop RA. Indeed this might even be anticipated to be more common than RA occurring first as the peak age of onset of RA is characteristically in the fourth and fifth decades, ${ }^{23}$ which is a decade older than that of $\mathrm{SLE}^{7}$. Should an equal number of patients have SLE and then RA, as have the converse, then some $7 \cdot 2 \%$ of our SLE patients would be predicted to have concurrent RA.

Further difficulty in making the dual diagnoses may arise because the conditions that allow the expression of one disease may mask the other. For example, oestrogens or pregnancy may improve $\mathrm{RA}^{24}$ while worsening or precipitating SLE. ${ }^{25}$ Similarly, it is possible that one disease may modify the other, and the fact that some of our patients either failed to develop erosions or had minor erosive changes after several years of RA supports this. Moreover, these patients may represent a more complex subgroup of autoimmune connective tissue disease, with episodes of specific syndromes occurring at separate times or concurrently, as nine patients had coexistent sicca syndrome, two had definite dermatopolymyositis, and five had antiENA. In the presence of such a clustering of syndromes it may be difficult to delineate individual diagnoses and instead patients may be classified as having a multisystem connective tissue disease. ${ }^{26}$

In conclusion, we believe that the concurrence of RA and SLE is more common than previously suggested. Although it is difficult to correct for selection bias in patient referrals to our hospital, our data, in association with the well described serological overlap, support the contention that the two diseases do coexist more often than expected by chance alone.

MGC is in receipt of the Frank G Spurway Scholarship of the Arthritis Foundation of Australia. Work done was in part supported by Sutton Rheumatism Research Funds, the Royal North Shore Hospital. We thank Mr R Money for the photography and Ms D Carey for secretarial assistance.

\section{References}

1 Fischman A S. Abeles M. Zanetti M. Weinstein A. Rothfield $\mathrm{NF}$. The coexistence of rheumatoid arthritis and systemic lupus erythematosus: a case report and review of the literature. $J$ Rheumatol 1981; 8: 405-15.

2 Hochberg M C. Adult and juvenile rheumatoid arthritis: current epidemiologic concepts. Epidemiol Rev 1981; 3: 27-44.

3 Mawson A R. Are rheumatoid arthritis and systemic lupus crythematosus inversely related diseảses? Med Hypotheses 1985; 18: $377-86$. 
4 Tan E M. Cohen A S. Fries J F. et al. The 1982 revised criteria for the classification of systemic lupus erythematosus. Arthritis Rheum 1982: 25: 1271-7.

5 Ropes M W. Bennett G A. Cobb S. Jacox R. Jessar R A. 1958 revision of diagnostic criteria for rheumatoid arthritis. Bull Rheum Dis 1958: 9: 175-6.

6 Dubois E L. Friou G J. Chandor S. Rheumatoid nodules and rheumatoid granulomas in systemic lupus erythematosus JAMA 1972; 220: 515-8.

7 Lee P, Urowitz M B, Bookman A A M, et al. Systemic lupus erythematosus. A review of 110 cases with reference to nephritis, the nervous system. infections, aseptic necrosis and prognosis. $Q J$ Med 1977; 46: 1-32.

8 Weissman B N, Rappoport A S. Sosman J L. Schur P H. Radiographic findings in the hands in patients with systemic lupus erythematosus. Radiology 1978; 126: 313-7.

9 Vivian Wells J, Webb J, Van Deventer M, et al. In vivo anti-nuclear antibodies in epithelial biopsies in SLE and other connective tissue diseases. Clin Exp Immunol 1979; 38: 424-35.

10 Webb J. Whaley K. Evaluation of the native DNA-binding assay for DNA antibodies in systemic lupus erythematosus and other connective tissue discases. Med J Aust 1974: 2: 324-8

11 Appel G B. Silva F G. Pirani C L. Meltzer J I. Estes D. Renal involvement in systemic lupus erythematosus (SLE). A study of 56 patients emphasizing histologic classification. Medicine (Baltimore) 1978; 57: 371-410.

12 Chalmers A. Thompson D. Stcin H E. Reid G. Patterson A C. Systemic lupus erythematosus during penicillamine therapy for rheumatoid arthritis. Ann Intern Med 1982: 97: 659-63.

13 Webb J, Pollard K M. Induction of DNA-antibodics by Dpenicillamine. Clin Exp Rheumatol 1985; 3: 213-9.

14 Bohan A. Peter J B. Polymyositis and dermatomyositis. N Engl $J$ Med 1975; 292: 344-7. 403-7.

15 Isenberg D A. Tookman A. White A G. Hoffbrand B I.
Mowbray J. Erosive rheumatoid arthritis co-existing with systemic lupus erythematosus. A report of a case also showing atlanto-axial subluxation. Clin Rheumatol 1982: 1: 216-20.

16 Bresnihan F P. Ansell B M. Rheumatoid arthritis followed by systemic lupus crythematosus. Proc $R$ Soc Med 1975; 68: 596-7.

17 Ragsdale C G. Petty R E. Cassidy J T. Sullivan D B. The clinical progression of apparent juvenile rhcumatoid arthritis to systemic lupus erythematosus. J Rheumatol 1980: 7: 50-5.

18 Esdaile J M. Danoff D. Rosenthall L. Gutkowski A. Deforming arthritis in systemic lupus erythematosus. Ann Rheum Dis 1981: 40: 124-6

19 Labowitz R. Schumacher H R. Articular manifestations of systemic lupus erythematosus. Ann Intern Med 1971: 74: 911-21.

20 Francis H. Kendrick J. Bell C. Webb J. Rheumatoid arthritisa review of 204 patients. Aust NZ J Med 1984; 14: 351A.

21 Baker S B. Rovira J R. Campion E W. Mills J A. Late onset systemic lupus erythematosus. Am J Med 1979; 66: 727-32.

22 Gossat D M. Walls R S. Systemic lupus erythematosus in later life. Med J Aust 1982; 1: 297-9.

23 Buchanan W W. Kean W F. Articular and systemic manifestations of rheumatoid arthritis. In: Scott J T. ed. Copeman's textbook of the rheumatic diseases. Edinburgh: Churchill Livingstonc. 1986: 653-705.

24 Cronin M E. Rheumatic aspects of endocrinopathies. In McCarty D J. ed. Arthritis and allied conditions. Philadelphia: Lea and Febiger, 1985: 1607-23.

25 Rothfield N. Clinical features of systemic lupus erythematosus. In: Kelley W N. Harris E D. Ruddy S. Sledge C B. eds. Textbook of rheumatology. 2nd ed. Philadelphia: Saunders. 1985: 1070-97.

26 Cohen M G. Ho K K. Webb J. Finger joint calcinosis followe by ostcolysis in a patient with multisystem connective tissue discase and anti-Jo-1 antibody. $J$ Rheumatol (in press). 